\title{
Submitted to

Self-suspended Nanomesh Scaffold for Ultrafast Flexible Photodetectors Based on

\section{Organic Semiconducting Crystals}

Lei Zhang, ${ }^{1,}{ }^{*}$ Nadiia Pastukhova, ${ }^{2}$ Yifan Yao, ${ }^{1}$ Xiaolan Zhong, ${ }^{1}$ Egon Pavlica, ${ }^{2}$ Gvido Bratina, $^{3}$ Emanuele Orgiu ${ }^{1, \pm} *$ \& Paolo Samorl ${ }^{*}$

${ }^{1}$ Dr. L. Zhang, Dr. Y. F. Yao, Dr. X. L. Zhong, Prof. E. Orgiu, Prof. P. Samorì

University of Strasbourg, CNRS, ISIS UMR 7006

8 allée Gaspard Monge, F-67000 Strasbourg, France

E-mail: samori@unistra.fr

${ }^{2}$ Dr. N. Pasthukova, Prof. E. Pavlica, Prof. G. Bratina

Laboratory of Organic Matter Physics, University of Nova Gorica

Vipavska 11c, SI-5270 Ajdovščina, Slovenia

$\S$ Present address: Key Laboratory of Mesoscopic Chemistry of MOE, School of Chemistry and Chemical Engineering, Nanjing University

Nanjing, Jiangsu 210023, China

E-mail: zhangleichem@nju.edu.cn

${ }^{ \pm}$Present address: Institut national de la recherche scientifique (INRS), EMT Center, 1650 Blvd. Lionel-Boulet, J3X 1S2 Varennes, Québec, Canada.

E-mail: emanuele.orgiu@emt.inrs.ca

Keywords: nanofabrication, optoelectronic devices, organic crystalline heterojunctions, $\pi$ conjugated materials 


\begin{abstract}
Self-standing nanostructures are of fundamental interest in materials science and nanoscience and are widely used in (opto-)electronic and photonic devices as well as in microelectromechanical systems. To date, large-area and self-standing nanoelectrode arrays assembled on flexible substrates have not been reported. Here we demonstrate the fabrication of a hollow nanomesh scaffold on glass and plastic substrates with a large surface area over 1 $\mathrm{mm}^{2}$ and ultra-low leakage current density $\left(\sim 1-10 \mathrm{pA} / \mathrm{mm}^{2} @ 2 \mathrm{~V}\right)$ across the empty scaffold. Thanks to the continuous sub-micrometer space formed in between the nanomesh and the bottom electrode, we could observe highly crystalline and dendritic domains of 6,13bis(triisopropylsilylethinyl)pentacene (TIPS-PEN) growing within the hollow cavity. The high degree of order at the supramolecular level leads to efficient charge and exciton transport; the photovoltaic detector supported on flexible PET substrates exhibit an ultrafast photoresponse time as short as $8 \mathrm{~ns}$ and a signal-to-noise ratio approaching $10^{5}$. Such a hollow scaffold holds great potential as a novel device architecture towards flexible (opto-)electronic applications based on self-assembled micro/nano-crystals.
\end{abstract}

Submitted to 
During the last three decades, organic semiconductors (OSCs) have been incorporated as active component into numerous optoelectronic devices thereby endowing to the latter attractive features such as solution processability, intrinsic flexibility and tunable properties by rational design of the molecular structures and their assemblies. ${ }^{[1]}$ While a high degree of order at the supramolecular level is crucial in order to harness various fundamental properties like charge transport, the generation of uniform films is key for large-area applications in (opto)electronics. Unfortunately, achieving simultaneously film crystallinity and uniformity for a given organic semiconductor is a greatest challenge. ${ }^{[2]}$ In order to maximize the degree of crystallinity, with the aim of generating high-quality discrete single crystals with perfect molecular packing, the nucleation barrier should be high enough to minimize the nucleation density. ${ }^{[3]}$ Conversely, morphologically uniform thin films are typically obtained by minimizing the nucleation barrier yielding polycrystalline or even amorphous structures during the aggregation process. ${ }^{[4]}$ In the case of a conventional photodiode or a solar cell in vertical geometry, ensuring the uniformity of the semiconductor layer is of paramount importance during fabrication because a pinhole-free film is vital to sustain a top electrode being deposited thereafter. In light of this requirement, the crystallinity in such an optoelectronic device has been largely sacrificed. Indeed, the best photovoltaic related (opto)electrical properties of organic semiconductors such as carrier mobility and exciton diffusion length have been recorded on single crystals or other highly ordered supramolecular assemblies. ${ }^{[5]}$ However, power conversion efficiency of photovoltaic devices based on those highly crystalline but less continuous assemblies still lags far behind, leaving plenty of room for future improvement. ${ }^{[6]}$

In a seminal work, bi-crystal p-n heterojunctions were integrated into photovoltaic detectors in a vertical and open device configuration where the uniformity of the active component could be disregarded since the work's focus was on spatially confined semiconducting crystals (Scheme 1). ${ }^{[7]}$ The nanomesh scaffolds used in such a proof-of- 


\section{Submitted to

concept study were fabricated on a standard Si wafer, thereby yielding non-flexible devices.

As shown in Scheme 1a, the nanomesh scaffold based on $\mathrm{Si}$ is composed of hundreds of separated cells being, arranged in a honeycomb fashion, which could wire-up supramolecular nanowires (Scheme 1b). Yet for hosting the growth of p-type organic crystals, the existence of $\mathrm{SiO}_{2}$ sidewall between neighboring cells could inhibit the further outspread crystallization once nucleation occurs inside individual cells, thus hindering the formation of crystalline domains extended over larger areas (Scheme 1c). In order to get rid of such physical impediment to the growth of extended semiconductor regions, it is highly desirable to connect adjacent cells to realize a large-area self-suspended nanomesh electrode.

As a major step forward, such a hollow nanomesh scaffold could be realized here on top of glass and plastic substrates instead of Si wafer (Figure 1). The fabrication procedure starts with spin-coating a reliable dielectric layer of cross-linked poly(4-vinylphenol) (CL-PVP) onto pre-patterned gold bottom electrode (Figure 1a). Since oxygen plasma can etch PVP, a very thin barrier layer ( $\sim 0.2 \mathrm{~nm}$ thick) of chromium was vacuum deposited on top of CL-PVP before self-assembly the polystyrene (PS) nanospheres into a hexagonally close-packed (hcp) monolayer. The nanomesh electrode was then patterned by evaporating a thin layer of gold (ca. $45 \mathrm{~nm}$ ) through the PS nanosphere mask, followed by the lift-off process (nanosphere lithography; NSL) ${ }^{[8]}$ As the last step to produce the hollow nanomesh scaffold, an oxygen plasma treatment was then employed to etch CL-PVP even underneath the nanomesh mask due to a transverse drilling effect. By controlling the duration of the over-etching, an array of CL-PVP nanopyramids was shaped to prop the hollow gold nanomesh up and prevent collapsing onto the bottom electrode, as illustrated in the scanning electron microscopic (SEM) image displayed in Figure $1 b$.

As shown in the last two diagrams of Figure 1a, the nanomesh structure acts as a crystal selector: PTCDI-C8 nanowire crystals, which have been grown in a solution by phaseexchange precipitation, are too large and stiff to pass through the mesh grid and so they could 
osited. In contrast, TIPS-PEN, which is molecularly dispersed in solution, could penetrate into the cavity between the top and bottom electrodes and seed in-situ the crystal formation. The interelectrodic junction, being empty, will be filled by TIPS-PEN crystals whose vertical size will be dictated by the thickness of the CL-PVP layer, and lateral size would extend over hundreds of micrometers, with the only boundaries being the fine CL-PVP pillars or nanopyramids remaining from the oxygen plasma treatment (Figure 1c). These TIPS-PEN crystals could also extend to the space underneath PTCDI-C8 crystalline nanowires (CNWs) to connect them to the bottom electrode by forming $\mathrm{p}-\mathrm{n}$ heterojunctions (Figure 1a and 1c). Significantly, such methodology to fabricate hollow nanomesh scaffold is also compatible with large-area plastic substrate of polyethylene terephthalate (PET; Figure 1d).

The self-suspended nanomesh scaffold can reach a high degree of structural uniformity over hundreds of micrometers thanks to unique capacity of PS nanospheres to assemble into hcp-packed monolayers (Figure 2a). A 45-nm thick gold nanomesh electrode was robust enough to sustain itself when CL-PVP has been partly removed from underneath (Figure $2 b$ ). A large statistics of leakage current upon DC bias was carried out and the results are summarized in Figure S1. We defined that a leakage current density of $1 \mathrm{nA} / \mathrm{mm}^{2} @ 0.5 \mathrm{~V}$ was indicative of a successful fabrication. On a device surface area of $1.0 \mathrm{~mm}^{2}, 42.7 \%$ and $83.3 \%$ of the devices comply with such threshold value, when supported on PET and glass substrates, respectively. As expected, a statistically increased occurrence of short circuits was measured when a thinner CL-PVP layer was employed (CL-PVP thickness being $460 \mathrm{~nm}$ on PET vs. $550 \mathrm{~nm}$ on glass). The successful fabrication of large-area hollow electrodes also represents a fundamental breakthrough in the field of nanotechnology considering that even a local defect/collapse of the nanostructures would lead to the short-circuit of the device as a whole. ${ }^{[9]}$ 


\section{Submitted to

Significantly, the obtained gold nanomesh was robust enough to support hundreds of nanowires per $\mathrm{mm}^{2}$ without collapsing (Figure 2c) while the leakage current could be kept at less than $0.1 \mathrm{nA} / \mathrm{mm}^{2} @ 2 \mathrm{~V}$ because the nanowires only made contact with the top electrode. As a control experiment, P3HT solution was drop-cast onto the nanomesh scaffold and it showed device current over 5 orders of magnitude greater when compared to that of PTCDIC8 CNWs, because molecularly dispersed P3HT solution could penetrate through the nanomesh into the space below and solidify, thus generating percolation pathways for charges to travel between the electrodes (Figure S1). In order to minimize such a redundant current pathway which does not flow across p-n heterojunctions, here a $150 \mathrm{~nm}$-thick lithium fluoride (LiF) was evaporated onto the hollow nanomesh exposing the PTCDI-C8 crystalline nanowires before growing TIPS-PEN crystals as displayed in Figure $2 \mathrm{~d}$ and $2 \mathrm{e}$. Such LiF layer results in an increased roughness of the top surface for both the gold and PTCDI-C8 CNWs, as portrayed in the SEM images.

Due to its dielectric nature, the LiF layer on the surface of top and bottom electrodes would inhibit TIPS-PEN to make electrical leakage sideways. Indeed, upon deposition of a LiF prepassivating layer we observed 2 orders of magnitude decrease in the current through TIPSPEN (Figure S1). During LiF evaporation, the existing PTCDI-C8 CNWs served as the shadow mask protecting the region just beneath from LiF coverage (Figure 2e). Due to such a self-alignment effect, the lower surface of PTCDI-C8 CNWs and the bottom electrode region just beneath are not electrically insulated. As shown in Figure $2 \mathrm{f}$ and $2 \mathrm{~g}$, TIPS-PEN molecules tend to nucleate and grow in the gap between two gold electrodes. Such a location is perfect for an efficient connection to the PTCDI-C8 nanowire lower surface as depicted in Figure $2 \mathrm{~h}$.

The p-n heterojunction between PTCDI-C8 and TIPS-PEN is the key interface for exciton dissociation, which is the origin of the photovoltaic effect. We should note that photovoltaic 
ectronic properties in view of the three effect is of utter importance amongst all the optoelectronic properties in view of the three following reasons: 1) As self-powered device, photovoltaic detector typically exhibits a large signal-to-noise ratio since the background current at $\mathrm{V}=0 \mathrm{~V}$ is ultralow in dark. ${ }^{[10]} 2$ ) Photovoltaic effect could be used to convert solar light into electricity for energy related applications. $\left.{ }^{[11]} 3\right)$ The short circuit current $\left(I_{\mathrm{SC}}\right)$ of a photovoltaic device could be regarded as the probe to the exciton behavior in OSCs by excluding any photoconduction (PC) effect. ${ }^{[12]}$

In this study, the photovoltaic properties of organic crystalline heterojunctions have been thoroughly investigated on both the pristine substrate as prepared at room temperature (Figure 3) and upon thermal annealing at $150{ }^{\circ} \mathrm{C}$ (Figure S2). In Figure 3a, the $I-V$ curves of the organic crystalline photodetector under light irradiation are displayed together with the curve recorded in the dark. The inset shows an unambiguous photovoltaic effect where the open-circuit voltage $\left(V_{\mathrm{OC}}\right)$ and the short-circuit current $\left(I_{\mathrm{SC}}\right)$ could be tuned upon varying the irradiation wavelength. The absolute value of the photocurrent was recorded and summarized into a 2D map by scanning both the applied voltage and the illumination wavelength (Figure $3 b)$. In such a contour image, the photocurrent minimum has portrayed the profile of $V_{\mathrm{OC}}$, which could be tuned from $40 \mathrm{mV}$ to $110 \mathrm{mV}$ by the irradiation wavelength. This phenomenon of wavelength-dependent $V_{\mathrm{OC}}$ could be explained by the fact that PTCDI-C 8 and TIPS-PEN are known to be sensitive to different wavelength bands. ${ }^{[7 \mathrm{~b}]}$

The color in the 2D map (Figure 3b) represents the absolute value of photocurrent, which also depends on the employed wavelength when the device was biased at a certain voltage. Since light source power also varies with the wavelength, we use photoresponsivity, $R$, as a function of wavelength to estimate the photocurrent contributions from each single component, i.e., PTCDI-C8 and TIPS-PEN. $R$ is obtained by dividing the device photocurrent, $I$, by the incident light power, $P$. As shown in Figure $3 \mathrm{c}$, the photoresponsivity $(R=I / P$; unit: $\mathrm{mA} / \mathrm{W}$ ) increases when a DC bias voltage is applied. We should note that all of the response 
shape in which the responsivity at long spectra curves at varying DC bias showed similar shape in which the responsivity at long wavelengths being located at the absorption peak of TIPS-PEN (ca. $650 \mathrm{~nm}$; red light) is more significant than previous results. ${ }^{[7 b]}$ This phenomenon indicates that the TIPS-PEN crystals start to contribute more to the photocurrent than PTCDI-C8 nanowires in the case of nonannealed crystals on hollow nanomesh scaffold. It is believed that a greater value of charge carrier mobility in a specific organic semiconductor indicates a higher degree of supramolecular order ${ }^{[1 \mathrm{~b}]}$ and likely leads to longer exciton diffusion lengths, ${ }^{[5 \mathrm{a}]}$ which would account to a more efficient generation of short circuit current. ${ }^{[12 \mathrm{~d}]}$ For example, $150{ }^{\circ} \mathrm{C}$ annealing for 1 hour would not only decrease the hole mobility in TIPS-PEN (Figure S3), but also degrade the photoresponsivity at $650 \mathrm{~nm}$ dramatically (Figure S2), meanwhile the photoresponsivity at PTCDI-C8 nanowire absorbance band becomes more significant upon annealing thanks to the enhanced mobility (see Figure S4 and S5).

For the sake of comparison, the photoresponsivity of PTCDI-C8/TIPS-PEN bi-crystal devices based on the Si-based nanomesh scaffold have also been studied by varying the thermal annealing temperature from $80{ }^{\circ} \mathrm{C}$ to $150{ }^{\circ} \mathrm{C}$ (Figure $\mathrm{S} 6, \mathrm{~S} 7$ and S8). The corresponding UV-vis absorbance of TIPS-PEN and PTCDI-C8 films are displayed in Figure S5. In order to compare the results obtained by using the different device configurations, we defined a parameter of $\left(N_{\mathrm{e}} / N_{\mathrm{ph}}\right)_{690} /\left(N_{\mathrm{e}} / N_{\mathrm{ph}}\right)_{500}$ to evaluate the contribution from PTCDI-C 8 and TIPS-PEN by calculating the ratio between $N_{\mathrm{e}} / N_{\mathrm{ph}}$ at $690 \mathrm{~nm}$ (only sensitive to TIPS-PEN) and $500 \mathrm{~nm}$ (more sensitive to PTCDI-C8) illumination, where $N_{\mathrm{e}}$ represents the photogenerated charge carriers being collected as the short circuit current, and $N_{\mathrm{ph}}$ indicates the corresponding photons reaching the device surface area. The higher value of such a ratio means TIPS-PEN contributes more photocurrent compared to PTCDI-C8. We found that the value increased from 2.1 to 4.5 if the measurements were carried out by using the hollow nanomesh scaffold instead of the Si-based one. This observation suggests that a major 
Submitted to

\section{ADVANCED}

contribution comes from the thicker TIPS-PEN layer $(550 \mathrm{~nm})$ that absorbs more sufficient light than that being sustained by Si-based nanomesh scaffold ( 100 nm).

To evaluate the potential of bi-crystals PV device as a fast response photodetector, the nonannealed devices were illuminated by $3 \mathrm{~ns}$ laser pulses $(\lambda: 300-820 \mathrm{~nm})$ and time-dependent photocurrent was measured for 1000 ns after the laser pulse. The data are summarized in the 2D map in Figure 3d. Thanks to the high charge carriers mobility of non-annealed TIPS-PEN (ca. $0.1 \mathrm{~cm}^{2} / \mathrm{Vs}$, as confirmed by OFET characterization) and the short interelectrodic distance (e.g., $550 \mathrm{~nm}$ for the glass sample), a shorter time is needed for photo-generated charge carriers to reach the collecting electrode. In this study, the response time of such a bi-crystal optoelectronic device (Figure 3c) was found being as low as $10 \mathrm{~ns}$, being much faster than those we previously obtained after annealing at $150{ }^{\circ} \mathrm{C},{ }^{[7 b]}$ thus representing the shortest response time ever reported on the organic crystal-based PV detectors. ${ }^{[13]}$

Another appealing feature for the hollow nanomesh scaffold is that it could also be fabricated on flexible substrates like PET (see the SEM image in Figure S9). Figure 4a shows the light-dark $I-V$ curves of the non-annealed bi-crystals heterojunctions which were connected by the nanomesh scaffold supported on a PET foil. The signal-to-noise ratio could reach $10^{4}-10^{5}$ in the PV mode. Because the PVP dielectric layer used in the case of PET substrate was slightly thinner (420 nm on PET vs. $550 \mathrm{~nm}$ on glass), such a flexible bi-crystal PV detector showed a faster photoresponse time being as low as $8 \mathrm{~ns}$. The calculated external quantum efficiencies, i.e., the ratio between the amount of generated charge carriers and incident photons, are summarized in Figure 4c. In accordance with the results obtained on the glass substrate, here TIPS-PEN also contributes the majority of the photocurrent since the EQE curve is almost in the same shape with TIPS-PEN absorption spectrum. The other results of optoelectrical characterization are reported in Figure S9. As displayed in Figure 4e and 4f, the bending fatigue test was also performed on the empty nanomesh scaffold (PET substrate) 
and the resulting flexible PV detector with a bending radius of $1 \mathrm{~cm}$. Although the photoresponsivity decreased slightly to $79 \%$ of its initial performance after 1000 bending events over a cylinder with a $\sim 2 \mathrm{~cm}$ diameter, we should stress that the nanostructure of hollow nanomesh scaffold was robust enough to retain ultralow leakage current through the bending test.

In summary, we have reported the fabrication of self-suspended nanomesh scaffold on glass and plastic substrates to enable the asymmetric connection of p-n crystal heterojunctions into high performance photovoltaic detectors. TIPS-PEN (p-type) and PTCDI-C8 (n-type) were chosen in this study according to their high tendency to crystallize in solution and their complementary absorption in the UV-visible range. As a typical macroscopic nanoelectrode, the nanomesh scaffold exhibited sub-micrometer interelectrodic distance in the vertical dimension yet extend over 1 millimeter in the lateral directions. For a specific photovoltaic detector, the photoresponse time is determined by the velocity of charge carriers (i.e., the mobility) and the distance for them to be transported from the sites where they are generated to the electrodes. By the aid of the hollow nanomesh scaffold, both factors could be maximized simultaneously to afford an ultrafast photovoltaic detector which could respond in less than $10 \mathrm{~ns}$ to light irradiation. Most importantly, further improvement is highly possible by either using organic crystals possessing higher charge carrier mobilities or shorter interelectrodic distance in the hollow nanomesh scaffold. The latter effort was absolutely feasible by choosing polystyrene spheres with smaller diameter and making use of polymeric insulator with higher dielectric and mechanical robustness. Our self-suspended nanomesh scaffold represents a powerful device architecture that can take full advantage of the unique properties of organic semiconducting crystals for future large-area and flexible optoelectronics applications.

\section{Experimental Section}




\section{Submitted to

Materials: PS nanospheres (diameter $800 \mathrm{~nm})$ monodispersed suspension (10 w\%, in water), lithium fluoride (99.995\%), PTCDI-C8 (98\%), TIPS-PEN (>99\%), Poly(4-vinylphenol) (PVP) (weight-average molecular weight; $\mathrm{Mw} \sim 25,000 \mathrm{~g} / \mathrm{mol}$ ) and the cross-linker, i.e., poly(melamine-co-formaldehyde) methylated (PMF) solution (average $\mathrm{Mn} \sim 432 \mathrm{~g} / \mathrm{mol}$ ) were all purchased from Sigma-Aldrich and used as received without further purification.

Substrate preparation: The substrates of glass or PET were thoroughly cleaned and dried in high purity nitrogen flow. The bottom $\mathrm{Au} / \mathrm{Cr}$ electrode (Au: $30 \mathrm{~nm}$; $\mathrm{Cr}: 2 \mathrm{~nm}$ ) was deposited onto substrate through thermal evaporation. Then a bicomponent solution of PVP and the cross-linker in propylene glycol monomethyl ether acetate (PGMEA) with weight ratio of 5:1 was spin-coated onto the substrate bearing bottom electrode (spin-rate: $5000 \mathrm{rpm}$ ). A thermal annealing at $80{ }^{\circ} \mathrm{C}$ of 1 hour was used to remove the solvent residue before $150{ }^{\circ} \mathrm{C}$ thermal cross-linking treatment of the PVP dielectric layer. The CL-PVP thickness is easy to tune by preparing PVP solution in different concentration when a fixed spin-rate was used. The dielectric constants of glass and PET are 6.5 and 3.1, respectively.

Organic semiconductors deposition: PTCDI-C8 nanowires could be physisorbed on the Au top electrode of the nanomesh with high density coverage by exploiting the water-assisted arrangement as we have previously reported. ${ }^{7}$ Differently from previous reports, here the wet substrates after fishing the nanowires monolayer from water surface were kept in ethanol atmosphere during water-drying-up owing to the high surface tension of pure water. In fact, in absence of a low surface-tension solvent such as ethanol, the residual water drops could destroy the hollow nanostructure. Then TIPS-PEN crystals were drop-cast from a solution in anisole $(25 \mathrm{mg} / \mathrm{ml})$ which was pre-saturated by PTCDI-C8 to minimize the possible dissolution of the PTCDI-C8 nanowires. ${ }^{7 \mathrm{~b}}$

Optoelectronic measurement: The optoelectronic characterization was performed in a glove box filled with nitrogen. We used Polychrome $V$ system as monochromatic light source, which was purchased from Till Photonics. The output power has been calibrated by PM100A 


\section{ADNANCE
MAAERRALS}

Power Meter from Thorlabs company. Keithley 2636A system sourcemeter was used to perform the electrical characterization.

Transient photoresponse characterization: The transient photoconductivity measurement is schematically presented in Figure S10. The laser pulse duration was of 3 ns and its repetition frequency was of $10 \mathrm{~Hz}$. All measurements were performed in a nitrogen atmosphere with $\mathrm{H}_{2} \mathrm{O}$ and $\mathrm{O}_{2}$ levels below $10 \mathrm{ppm}$. The bottom Au electrode was referred as an anode and was connected to zero potential. The top $\mathrm{Au}$ electrode - cathode - was at a constant electric potential of $V_{\mathrm{B}}$. The AC signal from the cathode, separated by a circuit comprising a capacitor and a coil (bias-T) was connected to a $2 \mathrm{GHz}$ current amplifier and a $2.5 \mathrm{GHz}$ digitizing oscilloscope, represented as a resistor $R$ in Figure S10. As a result, each light pulse, which reached the structure, photogenerated a current transient $I(\mathrm{t})$.

\section{Supporting Information}

Supporting Information is available online from the Wiley Online Library or from the author.

\section{Acknowledgements}

The technical help of the STnano cleanroom facility is gratefully acknowledged. This work was financially supported by EC through the ERC Proof-of-Concept project FlexNanoOLED (GA-766936) and the Marie Curie ITN project iSwitch (GA No. 642196), the Labex projects CSC (ANR-10-LABX-0026 CSC) and NIE (ANR-11-LABX-0058 NIE) within the Investissement d'Avenir program ANR-10-IDEX-0002-02, and the International Center for Frontier Research in Chemistry (icFRC), and by the Slovenian Research Agency, program P1-0055. N. P. acknowledges the Slovene Human Resources Development and Scholarship Fund. 
Submitted to

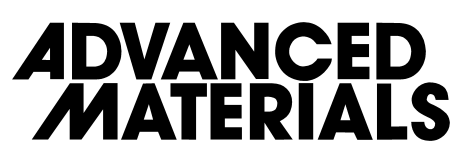

Received: ((will be filled in by the editorial staff))

Revised: ((will be filled in by the editorial staff))

Published online: ((will be filled in by the editorial staff))

[1] a) A. C. Arias, J. D. MacKenzie, I. McCulloch, J. Rivnay, A. Salleo, Chem. Rev. 2010, 110, 3; b) R. J. Li, W. P. Hu, Y. Q. Liu, D. B. Zhu, Acc. Chem. Res. 2010, 43, 529; c) A. Facchetti, Chem. Mater. 2011, 23, 733; d) R. J. Kumar, J. M. MacDonald, T. B. Singh, L. J. Waddington, A. B. Holmes, J. Am. Chem. Soc. 2011, 133, 8564; e) W. G. Zhu, R. H. Zheng, Y. G. Zhen, Z. Y. Yu, H. L. Dong, H. B. Fu, Q. Shi, W. P. Hu, J. Am. Chem. Soc. 2015, 137, 11038; f) F. X. Yang, S. S. Cheng, X. T. Zhang, X. C. Ren, R. J. Li, H. L. Dong, W. P. Hu, Adv. Mater. 2018, 30, 1702415.

[2] a) F. Heringdorf, M. C. Reuter, R. M. Tromp, Nature 2001, 412, 517; b) X. J. Zhang, J. S. Jie, W. Deng, Q. X. Shang, J. C. Wang, H. Wang, X. F. Chen, X. H. Zhang, Adv. Mater. 2016, 28, 2475; c) M. A. Fusella, F. Schreiber, K. Abbas, J. J. Kim, A. L. Briseno, B. P. Rand, Nano Lett. 2017, 17, 3040.

[3] a) A. L. Briseno, S. C. B. Mannsfeld, M. M. Ling, S. H. Liu, R. J. Tseng, C. Reese, M. E. Roberts, Y. Yang, F. Wudl, Z. N. Bao, Nature 2006, 444, 913; b) L. Q. Li, P. Gao, K. C. Schuermann, S. Ostendorp, W. C. Wang, C. A. Du, Y. Lei, H. Fuchs, L. De Cola, K. Müllen, L. F. Chi, J. Am. Chem. Soc. 2010, 132, 8807; c) K. S. Park, J. Baek, Y. Park, L. Lee, J. Hyon, Y. E. K. Lee, N. K. Shrestha, Y. Kang, M. M. Sung, Adv. Mater. 2017, 29, 1603285.

[4] a) J. E. Anthony, J. S. Brooks, D. L. Eaton, S. R. Parkin, J. Am. Chem. Soc. 2001, 123, 9482; b) R. Hamilton, J. Smith, S. Ogier, M. Heeney, J. E. Anthony, I. McCulloch, J. Veres, D. D. C. Bradley, T. D. Anthopoulos, Adv. Mater. 2009, 21, 1166; c) J. Smith, W. M. Zhang, R. Sougrat, K. Zhao, R. P. Li, D. K. Cha, A. Amassian, M. Heeney, I. McCulloch, T. D. Anthopoulos, Adv. Mater. 2012, 24, 2441. 


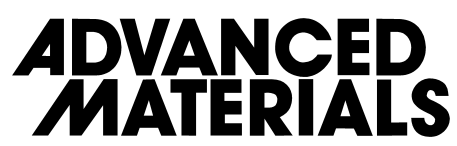

[5] a) H. Najafov, B. Lee, Q. Zhou, L. C. Feldman, V. Podzorov, Nat. Mater. 2010, 9, 938; b)

R. R. Lunt, J. B. Benziger, S. R. Forrest, Adv. Mater. 2010, 22, 1233; c) M. Sim, J. Shin, C.

Shim, M. Kim, S. B. Jo, J. H. Kim, K. Cho, J. Phys. Chem. C 2014, 118, 760; d) C. Wang, H.

Dong, L. Jiang, W. Hu, Chem. Soc. Rev. 2018, 47, 422; e) A. T. Haedler, K. Kreger, A. Issac,

B. Wittmann, M. Kivala, N. Hammer, J. Köhler, H. W. Schmidt, R. Hildner, Nature 2015, $523,196$.

[6] a) R. J. Tseng, R. Chan, V. C. Tung, Y. Yang, Adv. Mater. 2008, 20, 435; b) Y. J. Zhang, H. L. Dong, Q. X. Tang, S. Ferdous, F. Liu, S. C. B. Mannsfeld, W. P. Hu, A. L. Briseno, J. Am. Chem. Soc. 2010, 132, 11580; c) B. Verreet, P. Heremans, A. Stesmans, B. P. Rand, Adv. Mater. 2013, 25, 5504; d) H. Y. Li, C. C. Fan, W. F. Fu, H. L. L. Xin, H. Z. Chen, Angew. Chem. Int. Ed. 2015, 54, 956.

[7] a) L. Zhang, X. L. Zhong, E. Pavlica, S. L. Li, A. Klekachev, G. Bratina, T. W. Ebbesen, E. Orgiu, P. Samorì, Nat. Nanotech. 2016, 11, 900; b) L. Zhang, E. Pavlica, X. L. Zhong, F. Liscio, S. L. Li, G. Bratina, E. Orgiu, P. Samorì, Adv. Mater. 2017, 29, 1605760.

[8] T. C. Gao, B. M. Wang, B. Ding, J. K. Lee, P. W. Leu, Nano Lett. 2014, 14, 2105.

[9] a) Y. P. Zang, F. J. Zhang, D. Z. Huang, X. K. Gao, C. A. Di, D. B. Zhu, Nat. Comm. 2015, 6, 6269; b) L. Zhang, S. L. Li, M. A. Squillaci, X. L. Zhong, Y. F. Yao, E. Orgiu, P. Samorì, J. Am. Chem. Soc. 2017, 139, 14406.

[10] a) Q. H. Cui, L. Jiang, C. Zhang, Y. S. Zhao, W. P. Hu, J. N. Yao, Adv. Mater. 2012, 24, 2332; b) X. N. Sun, S. Vélez, A. Atxabal, A. Bedoya-Pinto, S. Parui, X. W. Zhu, R. Llopis, F. Casanova, L. E. Hueso, Science 2017, 357, 677.

[11] K. A. Mazzio, C. K. Luscombe, Chem. Soc. Rev. 2015, 44, 78.

[12] a) H. Alves, R. M. Pinto, E. S. Maçôas, Nat. Comm. 2013, 4, 1842; b) S. Karak, J. A. Lim, S. Ferdous, V. V. Duzhko, A. L. Briseno, Adv. Funct. Mater. 2014, 24, 1039; c) R. M. Pinto, W. Gouveia, E. M. S. Maçôas, I. C. Santos, S. Raja, C. Baleizão, H. Alves, ACS Appl. 
Mater. Interfaces 2015, 7, 27720; d) R. M. Pinto, E. M. S. Maçôas, A. I. S. Neves, S. Raja, C.

Baleizão, I. C. Santos, H. Alves, J. Am. Chem. Soc. 2015, 137, 7104.

[13] a) S. Ullbrich, B. Siegmund, A. Mischok, A. Hofacker, J. Benduhn, D. Spoltore, K. Vandewal, J. Phys. Chem. Lett. 2017, 8, 5621; b) J. Lee, S. R. A. Raza, P. J. Jeon, J. S. Kim, S. Im, NPG Asia Mater. 2016, 8, e278; c) Q. Burlingame, C. Coburn, X. Che, A. Panda, Y. Qu, S. R. Forrest, Nature 2018, 554, 77.

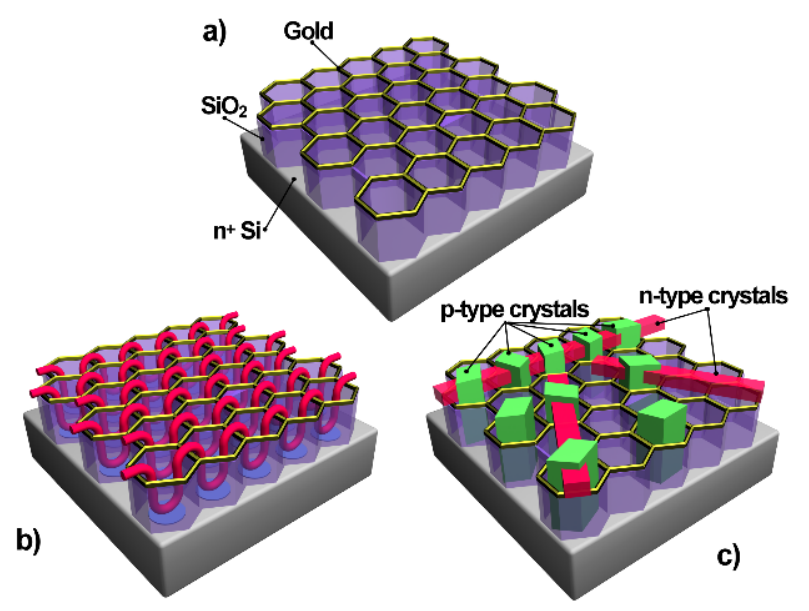

Scheme 1. Cartoon illustration in bird's-eye-view of a) An empty nanomesh scaffold; b) Supramolecular nanowires optoelectronic device, and c) Multiple bi-crystals photovoltaic device sustained by the nanomesh scaffold on Si wafer. 


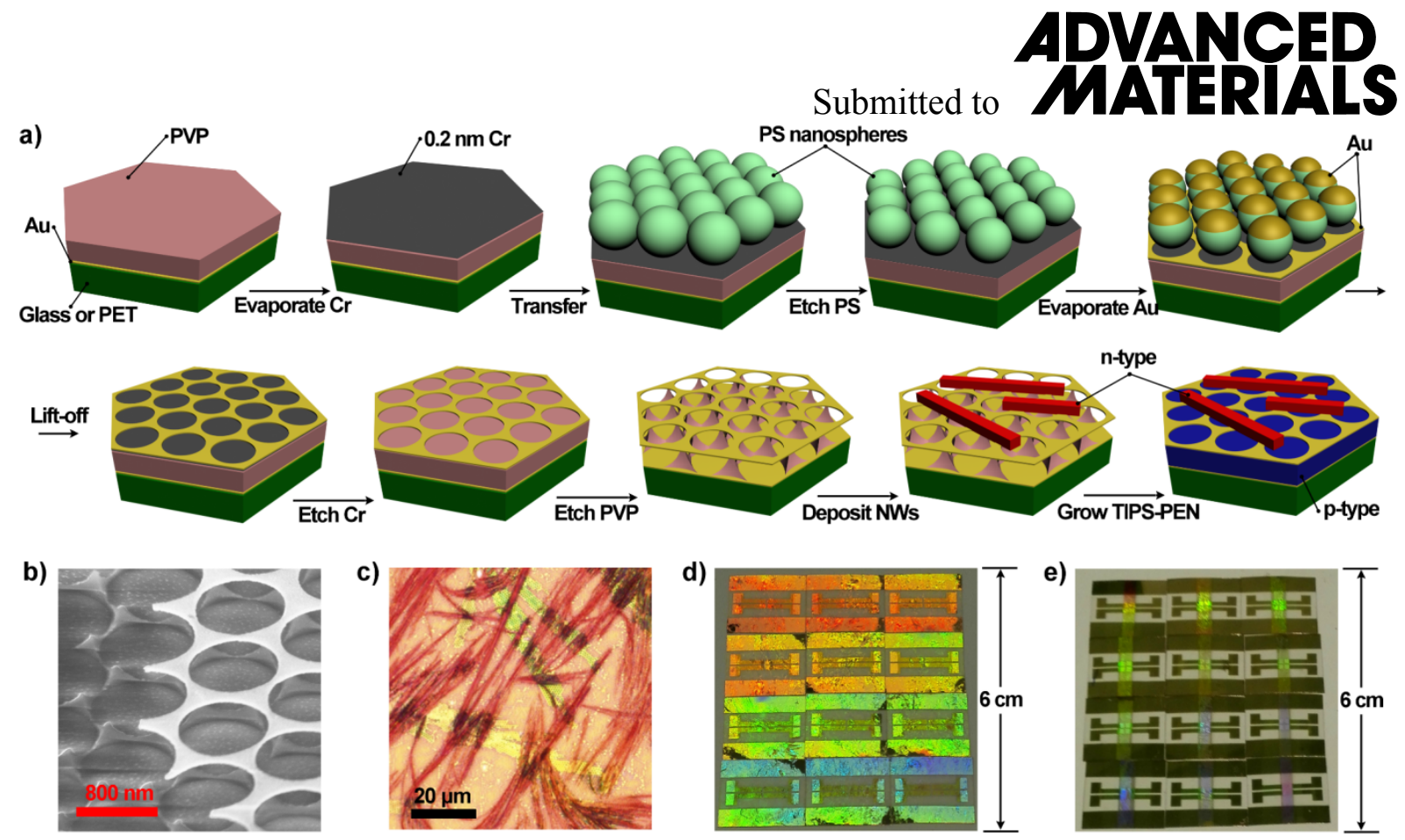

Figure 1. a) Schematic diagram showing the fabrication of self-suspended gold nanomesh

scaffold through nanospheres lithography (NSL) and oxygen plasma etching. Here PVP was chosen as the insulating layer due to its reliable dielectric property and its resist to common organic solvent after $150{ }^{\circ} \mathrm{C}$ cross-linking. The last cartoon showing multiple p-n heterojunctions sustained by the hollow nanomesh scaffold. b) SEM image of the bare suspending gold nanomesh (substrate tilt angle $=54^{\circ}$ ). NOTE: part of the nanomesh has been removed by scratching the device surface to clearly show the PVP nanopyramids array. c) Optical microscope picture illustrating the optoelectronic device being constituted of multiple PTCDI-C8 nanowires and large TIPS-pentacene crystalline domains. Photographs of PET plastic substrates $\left(6 \times 6 \mathrm{~cm}^{2}\right)$ bearing d) polystyrene nanospheres monolayer, and e) hollow nanomesh scaffold. 


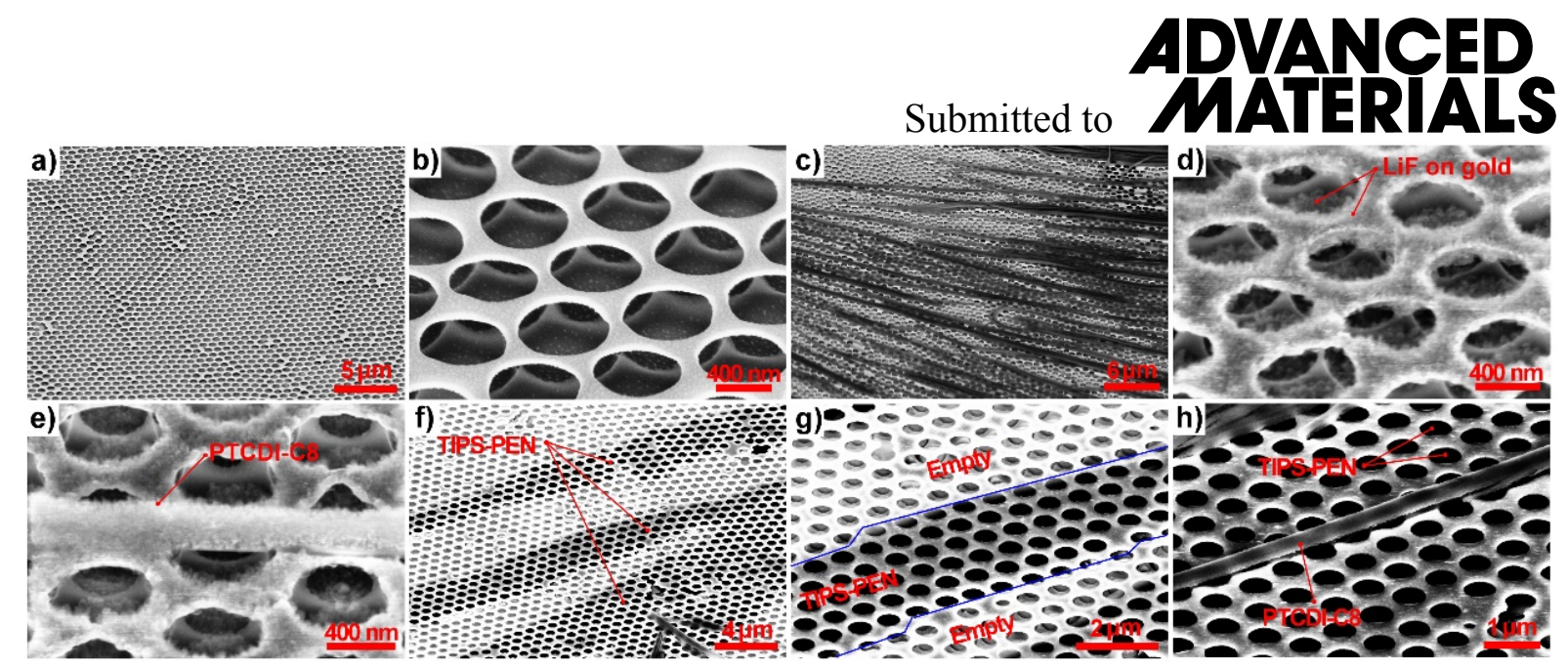

Figure 2. a) SEM images of the bare self-suspended nanomesh scaffold on glass substrate. b)

Zoom-in SEM image of the same sample in a). c) Multiple PTCDI-C8 crystalline nanowires (CNWs) on top of hollow gold nanomesh. d) A $150 \mathrm{~nm}$ thick lithium fluoride (LiF) layer is evaporated in vacuum to passivate the electrode surface and e) leave the bottom electrode beneath PTCDI-C8 CNWs still active thanks to the shadow mask effect of the CNWs. f) SEM image showing large TIPS-PEN crystalline domains grown in between top- and bottom electrodes by drop-casting. g) Zoom-in SEM image of the sample in f). h) SEM image illustrating organic crystalline an exemplary $\mathrm{p}-\mathrm{n}$ heterojunction sustained by the gold nanomesh scaffold. (all the above SEM images with the substrate tilt angle $=54^{\circ}$ ) 
a)
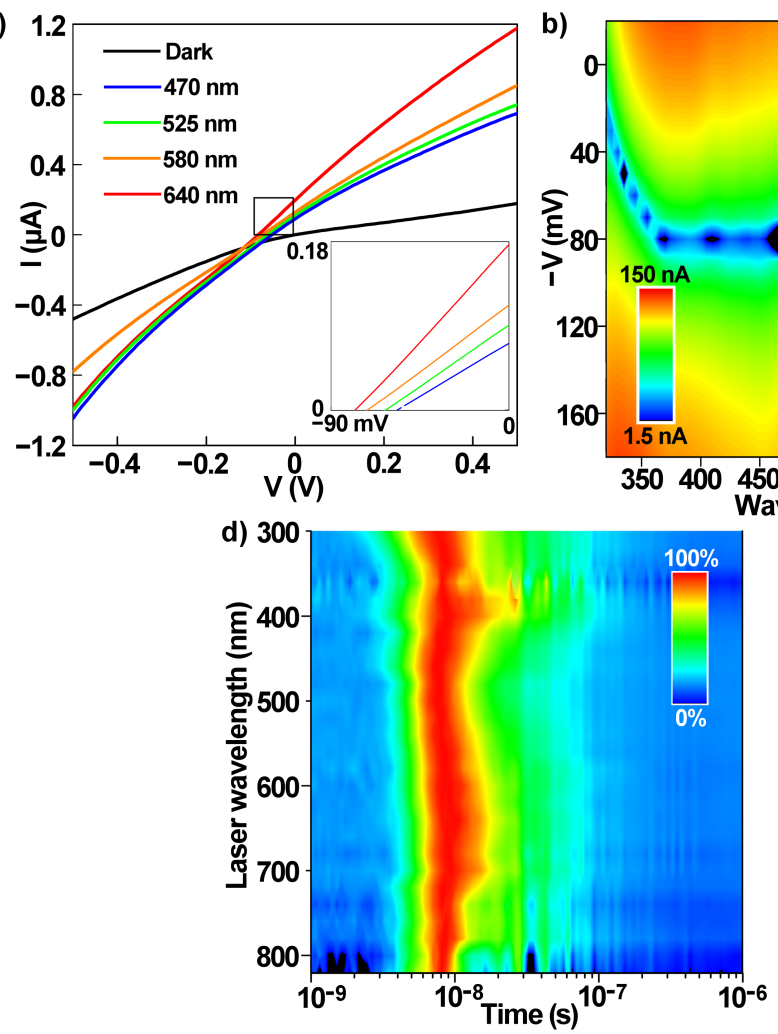

Submitted to
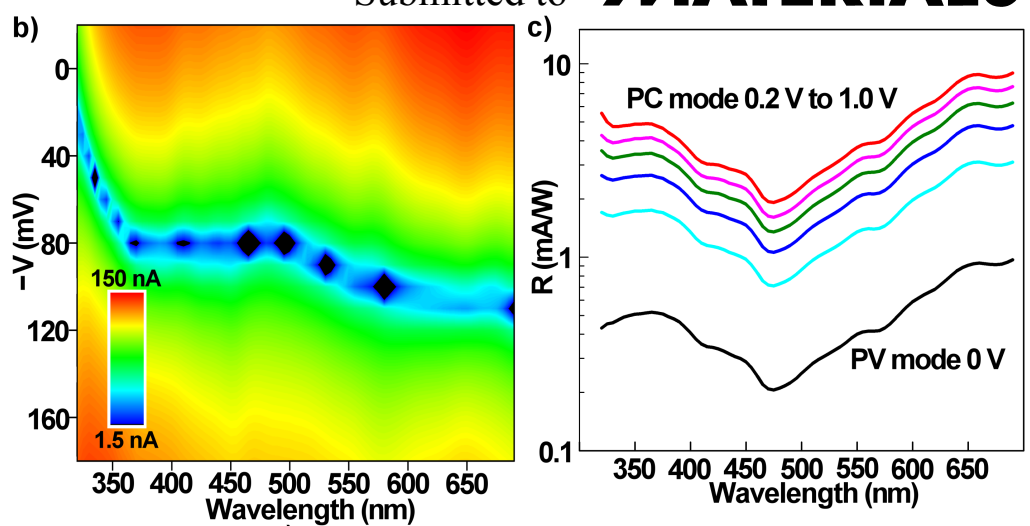

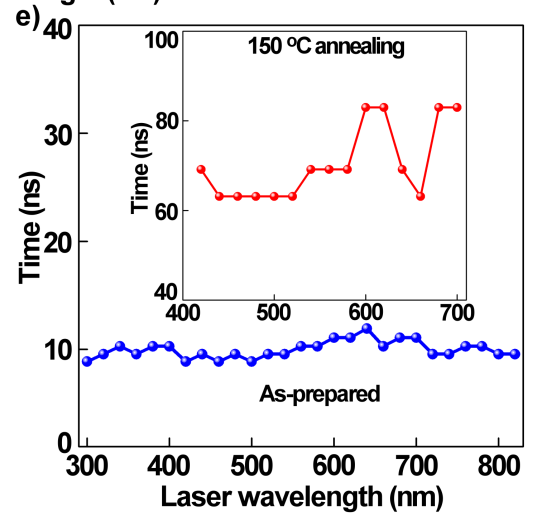

Figure 3. a) $I-V$ characteristics of the highly crystalline p-n heterojunctions in dark vs. upon irradiation at four different wavelenghts. Inset displays the zoom-in of a photovoltaic region with V scanning from $-100 \mathrm{mV}$ to $0 \mathrm{mV}$. [red light $640 \mathrm{~nm}, 0.166 \mathrm{~mW}$; yellow light $580 \mathrm{~nm}$, $0.209 \mathrm{~mW}$; green light $525 \mathrm{~nm}, 0.231 \mathrm{~mW}$; blue light $470 \mathrm{~nm}, 0.281 \mathrm{~mW}$. b) Twodimensional map of the photocurrent (absolute value) recorded by scanning the irradiation wavelength and applied voltage. c) Photoresponsivity spectra upon different voltage bias (photovoltaic mode: $0 \mathrm{~V}$; photovoltaic mode: $0.2 \mathrm{~V}$ to $1.0 \mathrm{~V}$ with an interval of $0.2 \mathrm{~V}$ ).

Photocurrent as a function of time after $3 \mathrm{~ns}$ laser pulse irradiation $(\lambda=300 \mathrm{~nm}$ to $820 \mathrm{~nm})$. e) The photoresponse time of the crystalline $\mathrm{p}$-n heterojunctions before and after $150{ }^{\circ} \mathrm{C}$ thermal treatment (inset of Fig. 3e). 

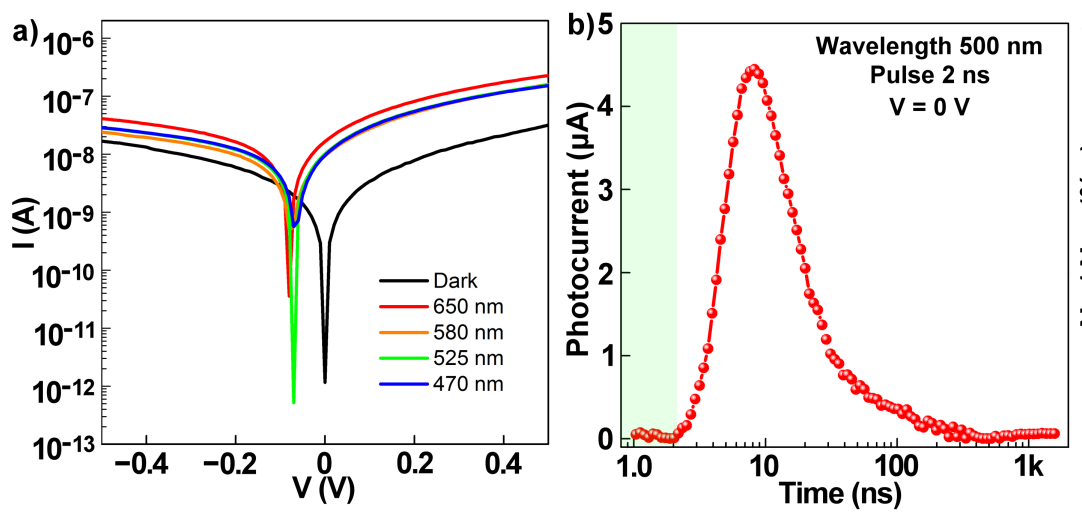

Submitted to

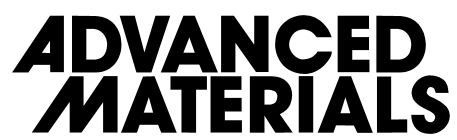

d)
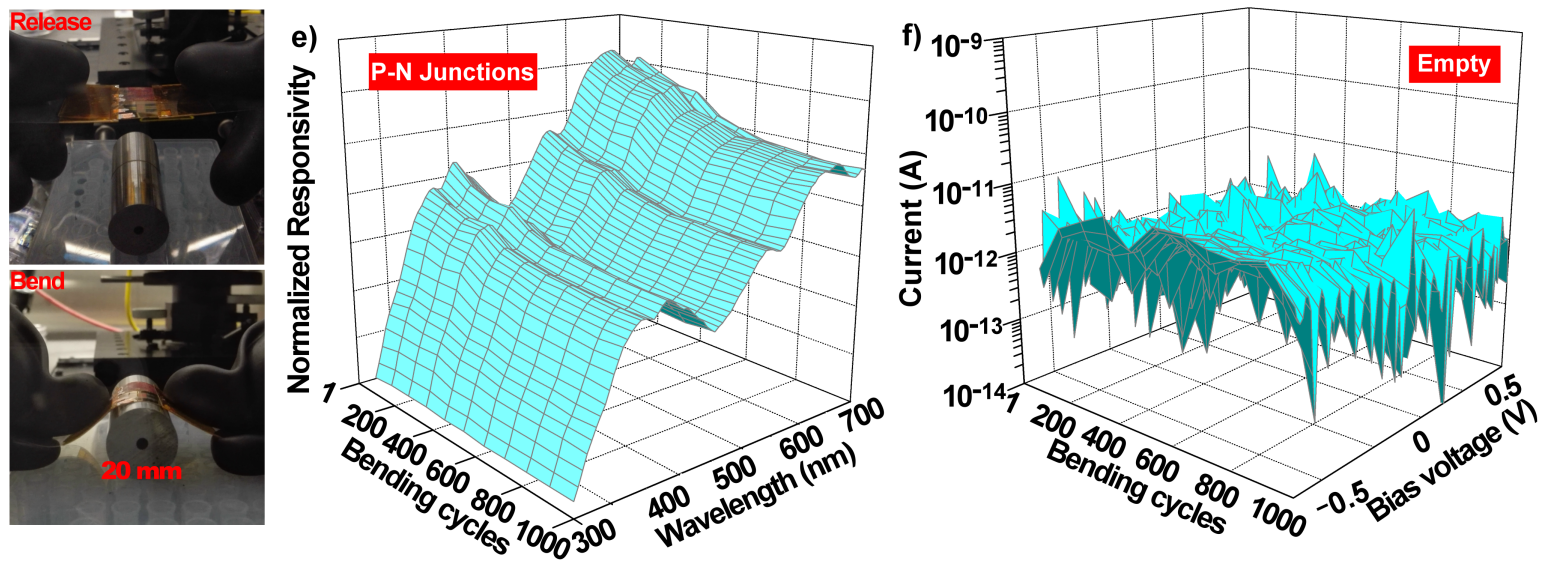

Figure 4. a) Dark and light $I-V$ curves of the flexible organic crystalline PV device in logarithmic coordinate. [red light $640 \mathrm{~nm}, 0.166 \mathrm{~mW}$; yellow light $580 \mathrm{~nm}, 0.209 \mathrm{~mW}$; green light $525 \mathrm{~nm}, 0.231 \mathrm{~mW}$; blue light $470 \mathrm{~nm}, 0.281 \mathrm{~mW}$. b) The transient photocurrent measurement by irradiating the device with 3 ns green laser pulse. c) Efficiency of the photons hitting the macroscopic device region to be converted into separated electron-holes pair. Normalized UV-vis absorption of PTCDI-C8 crystalline nanowires (CNWs) and dropcast TIPS-PEN polycrystalline film was drawn together. d) The bare self-suspended nanomesh scaffold and the resulting photovoltaic detectors were bent over a cylinder (bending radius of $1 \mathrm{~cm}$ ) for up to 1000 times. e) After 1000 times bending fatigue test, the photodetector showing the same photoresponsivity spectrum, but the absolute value degraded by $21 \%$. f) The hollow nanomesh scaffold would survive 1000 times bending fatigue test as evidenced by ultralow leakage current. 